\title{
Four-Wheeled Dead-Reckoning Model Calibration using RTS Smoothing
}

\author{
Anthony Welte, Philippe $\mathrm{Xu}$ and Philippe Bonnifait \\ Sorbonne universités, Université de Technologie de Compiègne, CNRS UMR 7253 Heudiasyc, France
}

\begin{abstract}
Localization is one of the main challenges to be addressed to develop autonomous vehicles able to perform complex maneuvers on roads opened to public traffic. Having an accurate dead-reckoning system is an essential step to reach this objective. This paper presents a dead-reckoning model for carlike vehicles that performs the data fusion of complementary and redundant sensors: wheel encoders, yaw rate gyro and steering wheel measurements. In order to get an accurate deadreckoning system with a drift reduced to the minimum, the parameters have to be well calibrated and the procedure has to be simple and efficient. We present a method able to accurately calibrate the parameters without knowing the ground truth by using a Rauch-Tung-Striebel smoothing scheme which enables to obtain state estimates as close to the ground truth as possible. The smoothed estimates are then used within a optimization process to calibrate the model parameters. The method has been tested using data recorded from an experimental vehicle on public roads. The results show a significant diminution of the dead-reckoning drift compared to a commonly used calibration method. We evaluate finally the average distance a vehicle can navigate without exteroceptive sensors by using the proposed four-wheeled dead reckoning system.
\end{abstract}

\section{INTRODUCTION}

Mobile robots can be qualified as autonomous if they are able to navigate in uncontrolled environments without the need for guidance devices. Alternatively, mobile robots can rely on guidance devices that allow them to travel a predefined navigation route in relatively controlled space. They are referred to as Autonomous Guided Vehicle (AGV) in this case. The same distinction can be made for autonomous cars. In order to develop truly "autonomous" vehicles, localization is an essential element for navigation, in particular, for planning and control. For that purpose, we believe that a strong dead-reckoning (DR) ability is mandatory. Indeed, while localization methods using exteroceptive sensors exist [5], [6], [14], they often only provide low-frequency and intermittent localization.

Many odometry methods exist in the literature. Methods using the vehicle on-board sensors have been used for many years[2]. These methods generally offer high frequency positioning but are limited by the accuracy of the sensors. More recently, methods using cameras [11], [7] and LiDAR [16] have been developed by estimating the relative displacement between two images or laser scans. However, since these methods use exteroceptive data, their accuracy depends on the quality of the observed environment. Thus, unstructured environments are challenging to such methods.

Localization using DR systems is subject to an unavoidable drift. This drift is firstly caused by the random-walk phenomenon that emerges from the integration of proprioceptive

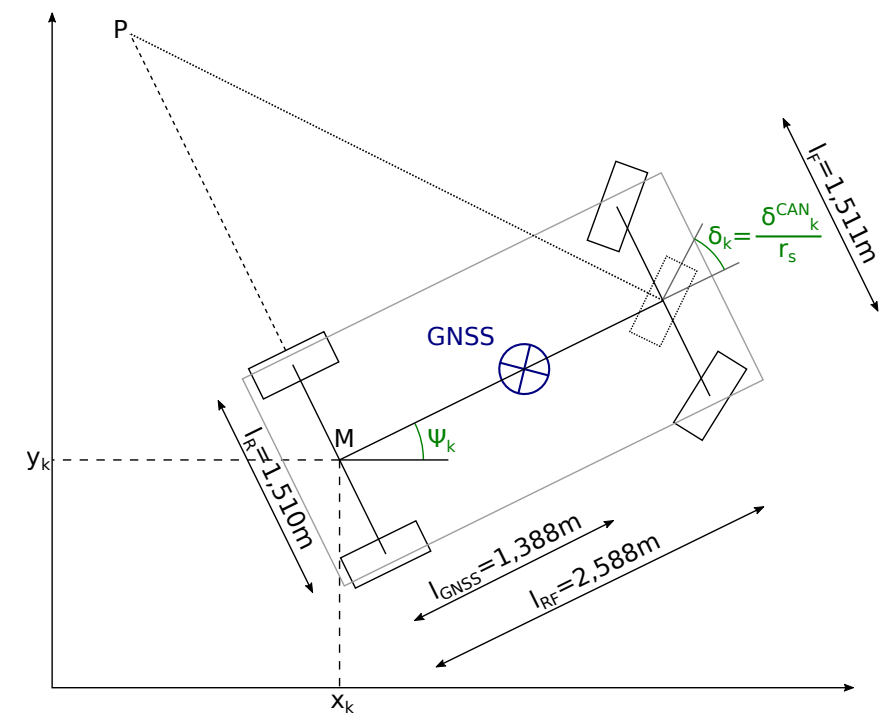

Fig. 1. Geometry of the experimental vehicle.

measurements over time and, secondly, by inaccuracies in the modeling of both the displacement of the mobile frame and the sensors observation models. The proprioceptive measurement errors are all the more significant that DR sensors are affected by systematic errors (biases/scaling factors), which increase the drift of the localization system.

In this work, we aim to obtain a DR model as accurate as possible using sensors readily available in production vehicles. To reach this objective, we first develop a model that uses all the different sensors knowing that they provide redundant information which is crucial to get an accurate and robust system able to handle wheel slippages or irregularities on the road surface. We then present a method to calibrate the most sensitive parameters in order to compensate the systematic errors of the sensors and observation models. For this, we have developed a calibration process which utilizes a Rauch-Tung-Striebel smoothing method to provide a better estimation of the vehicle state and then uses these states to calibrate the parameters of the system.

This paper is structured as follows: Section II details the DR model. The smoothing method is then explained in Section III followed in Section IV by the calibration method. Finally Section V presents the results obtained on experimental data. 


\section{ODOMETRIC MODEL}

\section{A. Evolution model}

In this work, we assume that the vehicle drives in a $2 \mathrm{D}$ environment. We therefore try to estimate at each step its position $\left[x_{k}, y_{k}\right]$ (point $\mathbf{M}$ in Fig. 1) and its yaw $\psi_{k}$. Additionally, the observations used in the fusion process depend on the vehicle speed $v_{k}$ and yaw rate $\dot{\psi}_{k}$. Therefore, the state $\boldsymbol{x}_{k}$ considered in the fusion process is 5-dimensional and is defined as follows:

$$
\boldsymbol{x}_{k}=\left[\begin{array}{lllll}
x_{k} & y_{k} & \psi_{k} & v_{k} & \dot{\psi}_{k}
\end{array}\right]^{T} .
$$

The state transition is defined with a non-linear function:

$$
\boldsymbol{x}_{k+1}=f\left(\boldsymbol{x}_{k}\right)+\boldsymbol{\alpha}_{k},
$$

where the $\boldsymbol{\alpha}_{k}$ is a zero mean Gaussian noise and $f$ the transition function is derived using Euler integration over a time $T_{e}$ as follows:

$$
\begin{aligned}
x_{k+1}=x_{k}+T_{e} \cdot v_{k} \cdot \cos \left(\psi_{k}+\frac{T_{e} \dot{\psi}_{k}}{2}\right) & & +\alpha_{k}^{x y} \\
y_{k+1}=y_{k}+T_{e} \cdot v_{k} \cdot \sin \left(\psi_{k}+\frac{T_{e} \dot{\psi}_{k}}{2}\right) & & +\alpha_{k}^{x y} \\
\psi_{k+1}=\psi_{k}+T_{e} \cdot \dot{\psi}_{k} & & +\alpha_{k}^{\psi} \\
v_{k+1}=v_{k} & & +\alpha_{k}^{v} \\
\dot{\psi}_{k+1}=\dot{\psi}_{k} & & +\alpha_{k}^{\dot{\psi}}
\end{aligned}
$$

It can be noted that instead of considering a constant yaw angle $\psi_{k}$ to compute the next position, a circular motion between $\psi_{k}$ and $\psi_{k+1}$ is considered. This can be approximated for small angle variations by using the angle $\psi_{k}+\frac{T_{e} \dot{\psi}_{k}}{2}$ instead of $\psi_{k}$.

As there is no prior information on the variation of the vehicle speed and yaw rate, the model considers them as constant and the noise model is chosen to reflect that lack of information. The selection of standard deviation is detailed in Section V.

\section{B. Observation model}

In order to reduce the natural drift that dead-reckoning processes are known for, the fusion of multiple sensors is performed. The measurements from a gyro, a speed sensor, four-wheel encoders as well as the steering wheel angle are used in the estimation process. These sensors operate at different frequencies $(50 \mathrm{~Hz}$ for the wheel encoders, $100 \mathrm{~Hz}$ for the rest) and are not synchronized. When these sensors are used in the fusion scheme, the state estimate is updated when the wheel encoder measurements are received. The most recent measurements of the other sensors are used in the fusion (half of the measurements from the other sensors are therefore discarded). We have observed experimentally that given the high frequency of the sensors and the relatively slow dynamic of the vehicle, interpolating the measurements does not lead to a significant accuracy improvement and is therefore not performed.
1) Gyro measurements: Most vehicles are equipped with a gyro that is used by the Electronic Stability Program (ESP) to prevent skidding. Automotive grade gyros are known to be affected by a significant bias denoted here $b_{\text {gyro }}$. Although this bias does not vary much during a normal driving sequence, it changes depending on environmental factors and therefore needs to be re-estimated at every vehicle start-up. Additionally, a zero-mean Gaussian noise $\beta_{k}^{\omega}$ affects the measurements. Its observation model can thus be written as

$$
\omega_{k}^{g y r o}=\dot{\psi}_{k}+b_{g y r o}+\beta_{k}^{\omega}
$$

where $\beta_{k}^{\omega}$ is a zero-mean Gaussian noise. In the rest of this section, a variable $\beta_{k}^{*}$ will refer to a zero-mean Gaussian noise.

In this model, we suppose that the gyro internally compensates the effect of the temperature.

2) Speed measurements: A speed measurement $v_{k}^{c a n}$ is available through the CAN bus of the vehicle. This measurement is most likely computed using the wheel encoders. It is however available at a higher frequency than the encoder measurements and does not seem correlated to those. Therefore, it can be used in the estimation. It is affected by a scaling factor $a_{v}$ that is particularly relevant at high speeds. The observation model is described as follows:

$$
v_{k}^{c a n}=a_{v} \cdot v_{k}+\beta_{k}^{v} .
$$

3) Wheel encoders: The wheel encoders provide the number of ticks $\Delta_{X X}{ }^{1}$ that occurred during a time $T(20 \mathrm{~ms}$ in our case). These measurements can be linked to the state variables using the Ackermann steering geometry and parameters such as the number of ticks per turn $N_{\text {wheel }}$, the wheel radii $\rho_{X X}$ and the front $\left(l_{F}\right)$ and rear $\left(l_{R}\right)$ track widths. Under the non-slippage assumption, the speed measured on each wheel of the vehicle can be linked to the vehicle longitudinal speed $v_{k}$ and its yaw rate $\dot{\psi}_{k}$ using geometrical considerations. Therefore, the number of ticks elapsed within a time interval $T$ can be linked to the vehicle state using the tick resolution $N_{\text {wheel }}$ and the wheel circumference as follows:

$$
\begin{aligned}
& \Delta_{k}^{R L}=\frac{N_{\text {wheel }}}{\rho_{R L}} T\left(v_{k}-\frac{l_{R}}{2} \dot{\psi}_{k}\right)+\beta_{k}^{R L} \\
& \Delta_{k}^{R R}=\frac{N_{\text {weel }}}{\rho_{R R}} T\left(v_{k}+\frac{l_{R}}{2} \dot{\psi}_{k}\right)+\beta_{k}^{R R} \\
& \Delta_{k}^{F L}=\frac{N_{w h e e l}}{\rho_{F L}} T \sqrt{l_{R F}^{2} \dot{\psi}_{k}^{2}+\left(v_{k}-\frac{l_{F}}{2} \dot{\psi}_{k}\right)^{2}}+\beta_{k}^{F L} \\
& \Delta_{k}^{F R}=\frac{N_{\text {weel }}}{\rho_{F R}} T \sqrt{l_{R F}^{2} \dot{\psi}_{k}^{2}+\left(v_{k}+\frac{l_{F}}{2} \dot{\psi}_{k}\right)^{2}}+\beta_{k}^{F R}
\end{aligned}
$$

Note that these measurements are affected by a quantization error due to the resolution of the sensors: $N_{w h e e l}$ ticks by turns (48 typically for a normal car, which is quite few compared to mobile robots encoders).

\footnotetext{
${ }^{1} X X$ refers interchangeably to: RL (Rear Left), RR (Rear Right), FL (Front Left), FR (Front Right)
} 
4) Steering wheel measurements: The steering wheel measurement $\delta_{k}^{C A N}$ is not used as is in the filter. Instead, it is expressed through the transversal speed of the front of the vehicle: $l_{R F} \cdot \dot{\psi}_{k}$. This is done to avoid having a prominent non-linear observation model at small speeds where this particular measurement is most useful. The steering wheel measurement is therefore scaled down by a factor $r_{s}$, which corresponds to the factor between the angle of the steering wheel and the angle of the virtual front wheel. The scaled measurement then corresponds to the angle of the virtual front wheel. Using this angle, we then know that $\tan \left(\frac{\delta_{k}^{C A N}}{r_{s}}\right)=\frac{l_{R F} \cdot \dot{\psi}_{k}}{v_{k-1}}$, therefore we obtain

$$
v_{k-1} \cdot \tan \left(\frac{\delta_{k}^{C A N}}{r_{s}}\right)=l_{R F} \cdot \dot{\psi}_{k}+v_{k-1} \cdot \beta_{k}^{\delta} .
$$

The dead reckoning observation model is thus as shown in equation (8)

The precision of some of these sensors can be improved by estimating some of their parameters with a calibration step. Therefore, the gyro bias, a scaling factor (for the speed measurement) and the wheel circumference are calibrated in order to have a better model of the sensors. From equation (8), we see that several parameters $\boldsymbol{p}=\left[p_{1}, \ldots, p_{6}\right]^{T}$ can be estimated in order for the model to best fit reality, namely

$$
\boldsymbol{p}=\left[\begin{array}{llllll}
b_{\text {gyro }} & a_{v} & 1 / \rho_{R L} & 1 / \rho_{R R} & 1 / \rho_{F L} & 1 / \rho_{F R}
\end{array}\right]^{T} .
$$

Note that to calibrate the wheel circumference $\rho_{X X}$, the inverse circumference $1 / \rho_{X X}$ is used instead which enables to solve the problems using Ordinary Least Squares.

\section{RAUCH-TUNG-STRIEBEL SMOOTHING}

\section{A. Global referencing}

In order to best calibrate the DR system, it is important to prevent drift on the trajectory used for calibration. Otherwise, the calibration parameters will be estimated to best match the drifting trajectory, and will therefore not be useful to limit the vehicle drift. For that purpose, we need external redundant information. A GNSS receiver is, therefore, used. The receiver is available at a lower frequency $(2 \mathrm{~Hz})$ and is used in a loose coupling scheme. The receiver antenna is located roughly mid way between the front and back wheel axle (see Fig. 1), thus a lever arm of length $l_{G N S S}$ has to be accounted for. The observation model of the receiver is given as follows:

$$
\begin{aligned}
& x_{k}^{G N S S}=x_{k}+l_{G N S S} \cdot \cos \left(\psi_{k}\right)+\beta_{k}^{G N S S} \\
& \underbrace{y_{k N S S}^{G}}_{\boldsymbol{z}_{k}^{G N S S}}=\underbrace{y_{k}+l_{G N S S} \cdot \sin \left(\psi_{k}\right)}_{\boldsymbol{h}_{k}^{G N S S}\left(\boldsymbol{x}_{k}, \boldsymbol{p}\right)}+\underbrace{\beta_{k N S S}^{G N S}}_{\boldsymbol{\beta}_{k}^{G N S S}} .
\end{aligned}
$$

The noise variance is provided by the GNSS receiver.

\section{B. Smoothing}

To estimate the calibration parameters, we need the most accurate state estimation we can obtain. For that purpose, conventional Kalman filtering suffer from the disadvantage of estimating states based only on current and past measurements. To exploit all the information that is available during the calibration stage, a smoothing step is applied after the filter and performed backward on the whole trajectory. The literature contains many smoothing methods[15], [1], [9]. In this paper we use the Rauch-Tung-Striebel smoother[13] (also known as Kalman smoother), since it is the simplest method and directly extends Kalman filtering.

To perform the forward processing of the smoothing, a Kalman filter is first ran using the available measurements. The equations for the filter are reminded next.

\section{Prediction:}

$$
\begin{aligned}
& \hat{\boldsymbol{x}}_{k \mid k-1}=\boldsymbol{f}_{k}\left(\hat{\boldsymbol{x}}_{k-1 \mid k-1}\right), \\
& \boldsymbol{P}_{k \mid k-1}=\boldsymbol{F}_{k} \boldsymbol{P}_{k-1 \mid k-1} \boldsymbol{F}_{k}^{T}+\boldsymbol{Q}_{k} .
\end{aligned}
$$

Update:

$$
\begin{aligned}
\tilde{\boldsymbol{y}}_{k} & =\boldsymbol{z}_{k}-\boldsymbol{h}_{k}\left(\hat{\boldsymbol{x}}_{k \mid k-1}\right), \\
\boldsymbol{S}_{k} & =\boldsymbol{H}_{k} \boldsymbol{P}_{k \mid k-1} \boldsymbol{H}_{k}^{T}+\boldsymbol{R}_{k}, \\
\boldsymbol{K}_{k} & =\boldsymbol{P}_{k \mid k-1} \boldsymbol{H}_{k}^{T} \boldsymbol{S}_{k}^{-1}, \\
\hat{\boldsymbol{x}}_{k \mid k} & =\hat{\boldsymbol{x}}_{k \mid k-1}+\boldsymbol{K}_{k} \tilde{\boldsymbol{y}}_{k}, \\
\boldsymbol{P}_{k \mid k} & =\left(\boldsymbol{I}-\boldsymbol{K}_{k} \boldsymbol{H}_{k}\right) \boldsymbol{P}_{k \mid k-1},
\end{aligned}
$$

where $\boldsymbol{H}_{k}$ is the Jacobian of the function $\boldsymbol{h}_{k}$ which either considers the DR measurements only:

$$
\boldsymbol{h}_{k}(\cdot)=\boldsymbol{h}_{k}^{D R}(\cdot)
$$

or both the GNSS and the DR measurements when they are available at the same time:

$$
\boldsymbol{h}_{k}(\cdot)=\left[\begin{array}{ll}
\boldsymbol{h}_{k}^{D R}(\cdot) & \boldsymbol{h}_{k}^{G N S S}
\end{array}\right]^{T} .
$$

During the filtering, several variables are recorded, namely the predicted state estimates $\left\{\hat{\boldsymbol{x}}_{k \mid k-1}\right\}_{k \in \llbracket 1, N \rrbracket}$ and their covariance matrices $\left\{\boldsymbol{P}_{k \mid k-1}\right\}_{k \in \llbracket 1, N \rrbracket}$ as well as the updated state estimates $\left\{\hat{\boldsymbol{x}}_{k \mid k}\right\}_{k \in \llbracket 0, N \rrbracket}$ and their covariance matrices $\left\{\boldsymbol{P}_{k \mid k}\right\}_{k \in \llbracket 0, N \rrbracket}$.

Smoothing. : The smoothing step is then performed backward, starting at the final state estimate $\hat{\boldsymbol{x}}_{N \mid N}$ back to state $\hat{\boldsymbol{x}}_{0 \mid N}$ as follows:

$$
\begin{aligned}
& \hat{\boldsymbol{x}}_{k \mid N}=\hat{\boldsymbol{x}}_{k \mid k}+\boldsymbol{J}_{k}\left(\hat{\boldsymbol{x}}_{k+1 \mid N}-\hat{\boldsymbol{x}}_{k+1 \mid k}\right), \\
& \boldsymbol{P}_{k \mid N}=\boldsymbol{P}_{k \mid k}+\boldsymbol{J}_{k}\left(\boldsymbol{P}_{k+1 \mid N}-\boldsymbol{P}_{k+1 \mid k}\right) \boldsymbol{J}_{k}^{T},
\end{aligned}
$$

where

$$
\boldsymbol{J}_{k}=\boldsymbol{P}_{k \mid k} \boldsymbol{F}_{k+1}^{T} \boldsymbol{P}_{k+1 \mid k}^{-1}
$$

After this process is done, we obtain smoothed state estimates $\left\{\hat{\boldsymbol{x}}_{k \mid N}\right\}_{k \in \llbracket 0, N \rrbracket}$ that are estimated knowing all the observations $\left\{\boldsymbol{z}_{k}\right\}_{k \in \llbracket 1, N \rrbracket}$. 


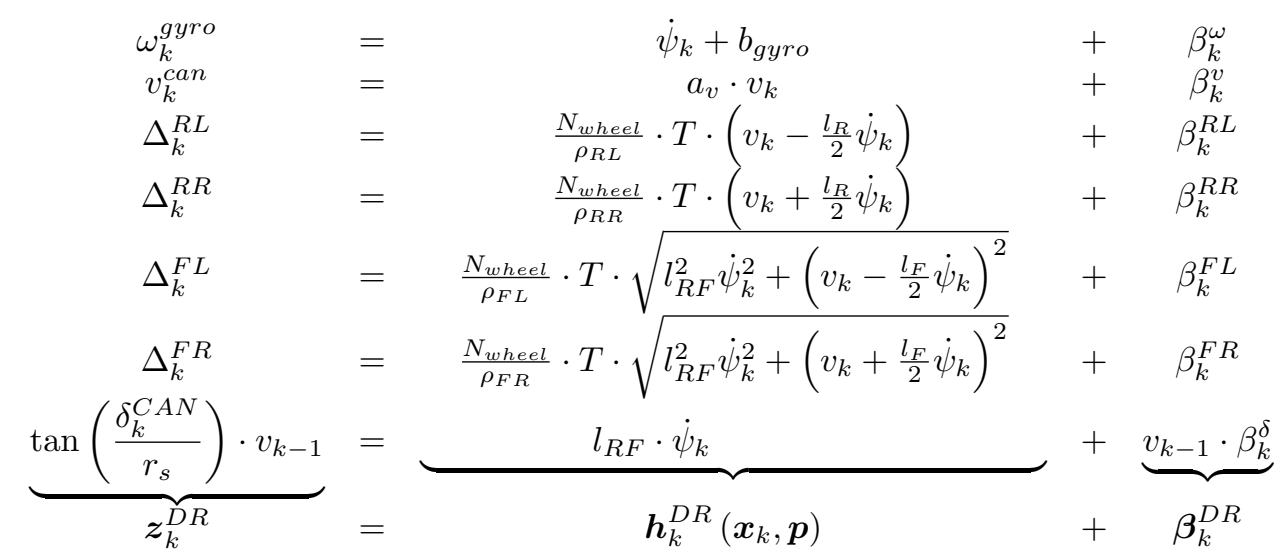

\section{PARAMETER CALIBRATION}

In order to limit the dead reckoning drift to a minimum, the calibration parameters need to be chosen so that systematic errors are reduced as much as possible.

The problem has been explored in the literature. Methods have been developed requiring the vehicle to perform particular trajectories [8], [3] to calibrate the sensors systematic errors. Others have used particle filtering [10] or augmented state Kalman filters [4] to find the model calibration parameters. Methods estimating the parameters in post-processing by comparing odometry and GNSS relative displacements have also been presented[12].

In this paper, we do not use a ground truth, nor an explicit calibration trajectory[3]. In practice, we record a first trajectory with all the raw data. This trajectory needs to be as diverse as possible in order to reach a well-conditioned calibration problem. As the ground truth is unknown, the smoothed states $\hat{\boldsymbol{x}}_{k \mid N}$ are used since they are as close to the ground truth as can be expected given the available observations. The problem is therefore a minimization problem and can be written as

$$
\hat{\boldsymbol{p}}=\underset{\boldsymbol{p}}{\operatorname{argmin}} \sum_{k=1}^{N}\left(\boldsymbol{z}_{k}-\boldsymbol{h}_{k}\left(\hat{\boldsymbol{x}}_{k \mid N}, \boldsymbol{p}\right)\right)^{2} .
$$

This minimization problem can be solved in our particular case using Least Squares because $\boldsymbol{h}_{k}$ is linear with respect to $\boldsymbol{p}$ for a given value of $\boldsymbol{x}_{k}$. Since each parameter $p_{i}$ appears in only one equation, the problem can be solved as multiple one-dimensional problems instead of one 6dimensional problem.

As such, the gyro bias is estimated by solving the following problem:

$$
\hat{p}_{1}=b_{\text {gyro }}=\underset{b_{\text {gyro }}}{\operatorname{argmin}} \sum_{k=1}^{N}\left(\omega_{k}^{\text {gyro }}-\hat{\dot{\psi}}_{k \mid N}-b_{\text {gyro }}\right)^{2} .
$$

The gyro bias can therefore be estimated simply as follows:

$$
b_{\text {gyro }}=\frac{1}{N} \sum_{k=1}^{N}\left(\omega_{k}^{\text {gyro }}-\hat{\dot{\psi}}_{k \mid N}\right) .
$$

The estimation of the speed scale factor $a_{v}$ is given as

$$
\hat{p}_{2}=a_{v}=\left(V^{T} V\right)^{-1} V^{T}\left[\begin{array}{c}
v_{1}^{C A N} \\
\vdots \\
v_{N}^{C A N}
\end{array}\right] \text {, }
$$

with $V=\left[\begin{array}{lll}\hat{v}_{1 \mid N} & \cdots & \hat{v}_{N \mid N}\end{array}\right]^{T}$.

The same estimation method is applied to estimate the $1 / \rho_{X X}$ parameters.

Remark 1. Note that there is no matrix inversion since the problems are one-dimensional. There is no numerical issues.

Remark 2. When estimating parameters that appear in equations containing the vehicle speed $v_{k}$, such as $a_{v}$ and $\rho_{X X}$, the epochs when the vehicle is slow (below $2 \mathrm{~m} / \mathrm{s}$ in our experiments) have to be discarded since some parameters become non-observable.

Remark 3. The smoothing and calibration processes can be iterated to reach higher accuracy.

\section{EXPERIMENTAL RESULTS}

\section{A. Experimental setup}

The method has been tested using one of the Heudiasyc laboratory experimental vehicles (see figure 2). It is set up with the Robot Operating System (ROS) middleware with which we are able to retrieve the sensors data with an accurate time-stamping. The DR sensors (yaw rate, speed, wheel top, steering angle) from the Anti-lock braking system (ABS) and Electronic Stability Program (ESP) of the car are available through a CAN-bus interface. The vehicle is also equipped with a non-differential u-blox M8T receiver set up to use GPS and GLONASS constellations. This receiver shares its antenna with a high accuracy Novatel SPAN-CPT that combines IMU, GNSS and RTK corrections to generate an accurate localization solution used as ground truth (GT) to evaluate our algorithms.

The aforementioned method has been tested using data recorded on public roads outside the laboratory. Several trajectories have been recorded in order to calibrate the dead reckoning model and test it on different data. In particular, two trajectories have been used to produce the experimental results. The first consists of a $4 \mathrm{~km}$ loop driven twice (dashed line on Fig. 3) adding up to an $8 \mathrm{~km}$ dataset. Once the 


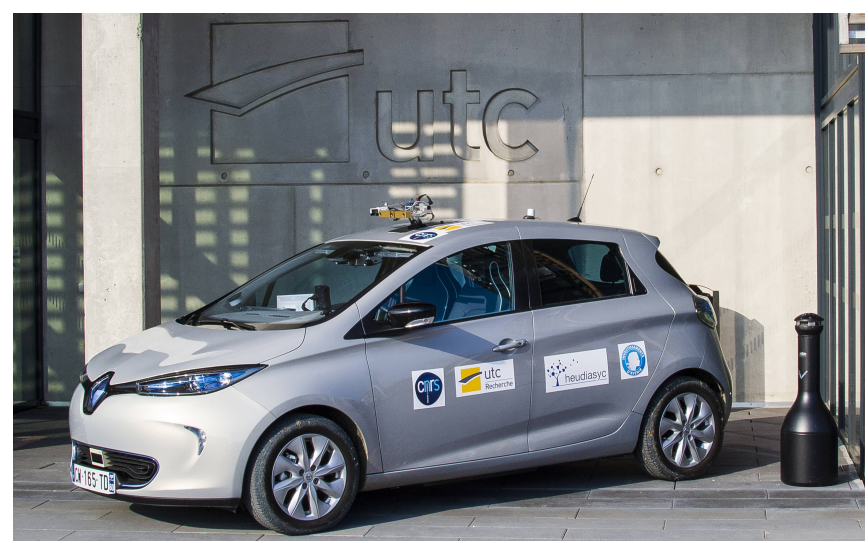

Fig. 2. Experimental vehicle of the Heudiasyc laboratory used in the experiments.

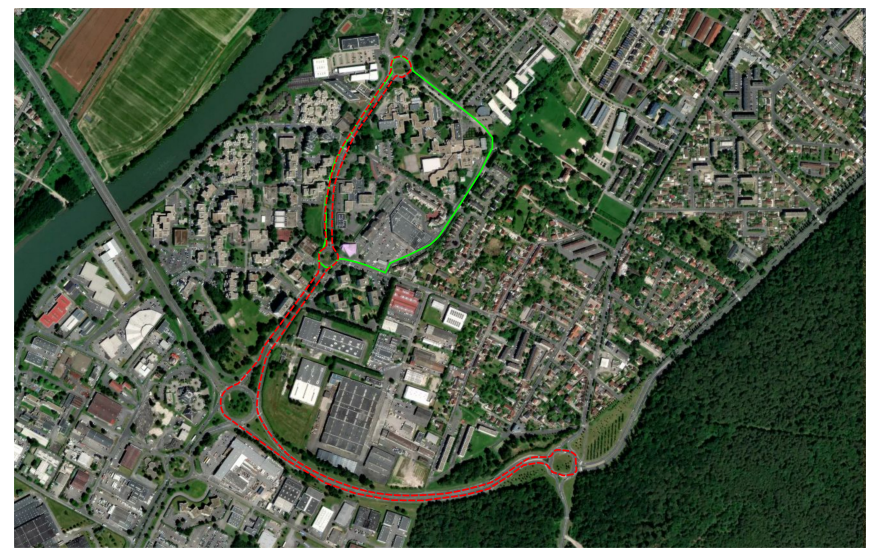

Fig. 3. Trajectories driven during the experiments. The route used for calibrating the odometric model parameters consists in two turns around the red trajectory (dashed line). The evaluation route consists of three turns around the green trajectory (solid line).

parameters have been calibrated using this trajectory, the dead reckoning has been tested without GNSS using a $1.7 \mathrm{~km}$ loop (solid line on Fig. 3) driven three times adding up to over $5 \mathrm{~km}$. The vehicle speed averaged about $30 \mathrm{~km} / \mathrm{h}$ and never went above $70 \mathrm{~km} / \mathrm{s}$ on both trajectories. The two trajectories have been recorded back-to-back meaning that the environmental condition can be assumed to be similar, in particular concerning the visibility of satellite constellations.

\section{B. Filter tuning}

The standard deviation of the evolution model noise is detailed in Table I. The standard deviation of the position and orientation have been set in order for the estimation to be consistent (i.e., the state estimate error stays bounded by $3 \sigma$ with a confidence rate of $99.7 \%$ ), whereas the standard deviation for the speed and the yaw rate have been set to fairly high values since we have no a priori knowledge about the evolution of these variables.

To set the measurement noise characteristics, the sensors error variances have been quantified using a ground truth equipment and the noise parameters have been set to match best the observed error distributions. Using the ground truth to set these parameters is not an issue since they are not
TABLE I

EVOLUTION MODEL NOISE CHARACTERISTICS

\begin{tabular}{|c|c|}
\hline Model noise & Standard deviation \\
\hline \hline$\alpha_{k}^{x y}$ & $10^{-3} \mathrm{~m}$ \\
\hline$\alpha_{k}^{\psi}$ & $10^{-5} \mathrm{rad}$ \\
\hline$\alpha_{k}^{v}$ & $10 \mathrm{~m} / \mathrm{s}$ \\
\hline$\alpha_{k}^{\psi}$ & $1 \mathrm{rad} / \mathrm{s}$ \\
\hline
\end{tabular}

TABLE II

OBSERVATION MODEL NOISE CHARACTERISTICS

\begin{tabular}{|c|c|}
\hline Model noise & Standard deviation \\
\hline$\beta_{k}^{\omega}$ & $0.003 \mathrm{rad} / \mathrm{s}$ \\
\hline$\beta_{k}^{v}$ & $10^{-1} \mathrm{~m} / \mathrm{s}$ \\
\hline$\beta_{k}^{R L}$ & 1 (unitless) \\
\hline$\beta_{k}^{R R}$ & 1 (unitless) \\
\hline$\beta_{k}^{H L}$ & 1 (unitless) \\
\hline$\beta_{k}^{H R}$ & 1 (unitless) \\
\hline$\beta_{k}^{\delta}$ & 0.02 (unitless) \\
\hline
\end{tabular}

expected to change over time and could be obtained from the sensors datasheet. The list of standard deviation is given in Table II.

\section{Results of the parameter calibration}

The parameters calibration has been performed using the method described in the previous sections and compared to the well known Zero Velocity Update (ZUPT) method. The method described in this paper uses the smoothed estimates as substitutes for the ground truth. We therefore also compare it to a calibration where the ground truth is available. The ZUPT method estimates the yaw rate bias by averaging the yaw rate measurements when the vehicle is stopped and therefore not turning. The other parameters, speed scale factor and wheel circumferences are set at their nominal values (1.0 for the speed scale factor, $1.92 \mathrm{~m}$ for the wheel circumferences). Table III details the parameters estimated using the two methods. The estimated gyro bias is noticeably different between our method and the ZUPT technique. It can be noted that estimating the gyro bias by comparing the measurements to those of an high accuracy IMU yield a bias of $-0.00298 \mathrm{rad} / \mathrm{s}$ on the calibration trajectory and -0.00269 $\mathrm{rad} / \mathrm{s}$ on the testing trajectory. This method therefore manages to estimate the yaw rate bias within a $7 \%$ margin of error which is vastly better than by using the ZUPT technique (error of 35\%). Regarding the speed scale factor and the wheel circumferences, the result are very close to the default values. This can be explain by the fact that the experimental vehicle is driven very little compared to personal vehicles. Hence, the wheels are not worn out.

To evaluate the quality of the calibration process and the performance of the dead reckoning method, we have carried out other experiments using a different, $5 \mathrm{~km}$ long, testing trajectory. The state has been initialized using the ground truth.

We use the following methodology to evaluate the drift. When a DR distance of $100 \mathrm{~m}$ has been traveled, the difference between the estimated position and the ground 
TABLE III

PARAMETERS CALIBRATION RESUlTS (ZUPT IS USED TO CALIBRATE $b_{\text {gyro }}$ ONLY, THE OTHER PARAMETERS ARE THE DEFAULTS).

\begin{tabular}{|l|c|c|c|c|c|c|}
\hline Method & $\begin{array}{c}b_{\text {gyro }} \\
(\mathrm{rad} / \mathrm{s})\end{array}$ & $a_{v}$ & $\begin{array}{c}\rho_{R L} \\
(\mathrm{~m})\end{array}$ & $\begin{array}{c}\rho_{R R} \\
(\mathrm{~m})\end{array}$ & $\begin{array}{c}\rho_{F L} \\
(\mathrm{~m})\end{array}$ & $\begin{array}{c}\rho_{F R} \\
(\mathrm{~m})\end{array}$ \\
\hline \hline GT & -0.00298 & 0.987 & 1.9166 & 1.9164 & 1.9134 & 1.9129 \\
\hline Ours & -0.00279 & 1.0 & 1.9198 & 1.9197 & 1.9177 & 1.9159 \\
\hline ZUPT & -0.00194 & 1.0 & 1.92 & 1.92 & 1.92 & 1.92 \\
\hline
\end{tabular}

TABLE IV

DEAD RECKONING DRIFT USING DIFFERENT CALIBRATION METHODS.

\begin{tabular}{|c|c|c|}
\hline Method & $\begin{array}{c}\text { position } \\
\text { mean error }\end{array}$ & $\begin{array}{c}\text { position error } \\
\text { standard deviation }\end{array}$ \\
\hline \hline GT & $0.79 \%$ & $0.39 \%$ \\
\hline Our method & $0.81 \%$ & $0.51 \%$ \\
\hline ZUPT & $0.93 \%$ & $0.57 \%$ \\
\hline
\end{tabular}

truth is computed and recorded. The state is then corrected using the ground truth and the process continues. The quality of the dead reckoning is evaluated as the average distance drifted per $100 \mathrm{~m}$. The results of the experiments are reported in table IV and the error distributions are shown in Fig. 4.

In the context of autonomous driving it is also useful to know how long one can drive using DR without going outside of the lane. To evaluate this we apply a similar strategy than previously but this time the estimation is not corrected every $100 \mathrm{~m}$ but only when the estimation error reaches $1 \mathrm{~m}$. The result of this test is shown in Fig. 5 . The proposed method increases quite significantly the DR navigation performance. On this experiment, we estimate that the car is able to maintain a 1-meter accuracy at least during 100 meters and sometimes it can be more than 200 meters. It should be noted that since the estimation is corrected only once the $1 \mathrm{~m}$ threshold is reached, the epochs at which the corrections happen are different for the two methods. This results in DR starting at different epochs and so comparing the results is difficult.

The trajectories obtained by the different parameter calibration methods are compared in Fig. 6. Both ZUPT and our method are significantly better than using standard parameters. This highlight the importance of calibrating the sensors. Our method performs slightly better than ZUPT which is most likely due to the better estimation of the gyro bias.

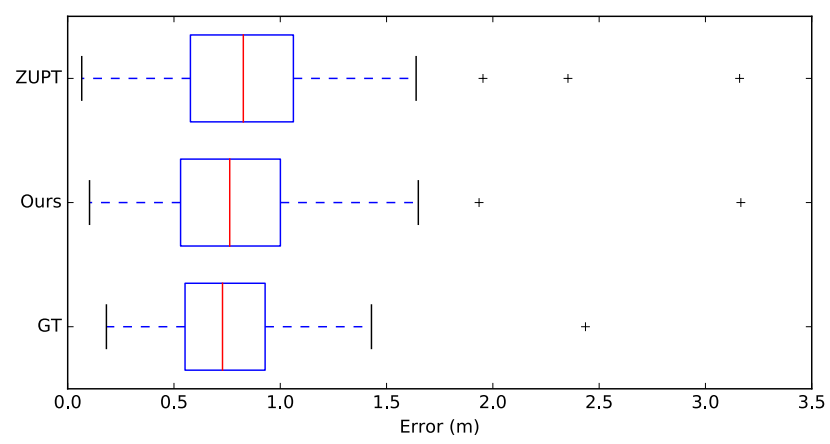

Fig. 4. Horizontal error distribution after $100 \mathrm{~m}$ traveled in DR.

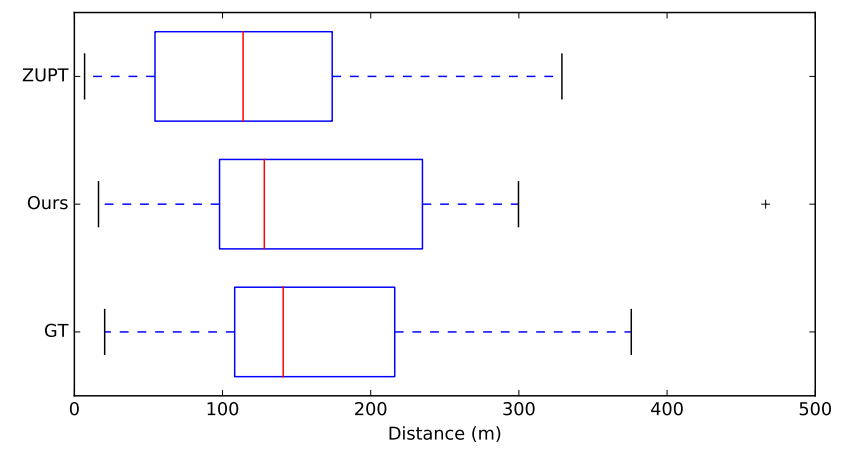

Fig. 5. Distance traveled in DR before reaching an error of one meter.

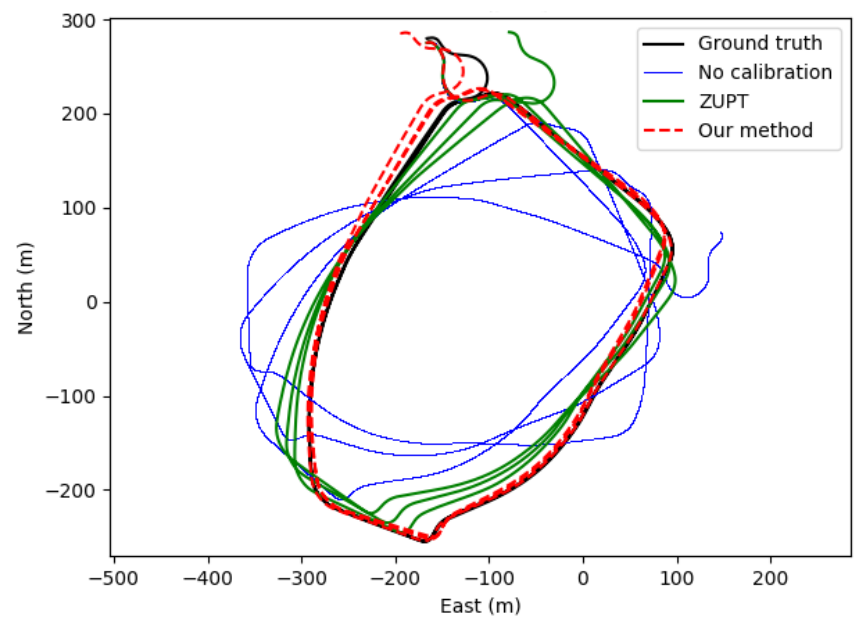

Fig. 6. Dead-reckoning results with different calibration methods.

\section{CONCLUSION}

This paper has presented a dead-reckoning model that fuses all the sensors usually available on modern cars. This methods merges redundant sensors and therefore is more robust that classical methods that usually use only the wheel speed sensors of the rear wheels. We have presented a new calibration method that is easy to implement. By performing smoothing over a calibration trajectory, parameters compensating for the sensors systematic errors are well estimated. The resulting model is more accurate, therefore its drift is reduced. Results obtained using experimental data obtained on public road shows a clear improvement compared to a non calibrated system or a method that uses the ZUPT method when the car is motion less. We have also evaluated the accuracy of a well calibrated model. Based on our experimental results, we estimate that the drift remains lower than 1-meter up to 100 meters of DR navigation which is very interesting for many functions that rely on localization for autonomous driving.

\section{ACKNOWLEDGMENT}

This work is carried out within SIVALab, a shared laboratory between Renault and Heudiasyc (UTC/CNRS), and cofinanced by the Hauts-de-France region (ERDF grant) and Renault. 


\section{REFERENCES}

[1] G.J. Bierman. Factorization Methods for Discrete Sequential Estimation, volume 128. 1977.

[2] P. Bonnifait, P. Bouron, P. Crubille, and D. Meizel. Data fusion of four ABS sensors and GPS for an enhanced localization of carlike vehicles. In IEEE International Conference on Robotics and Automation (ICRA). IEEE, 2001.

[3] J. Borenstein and L. Feng. Correction of systematic odometry errors in mobile robots. In IEEE International Conference on Intelligent Robots and Systems (IROS), pages 569-574, Aug 1995.

[4] A. Brunker, T. Wohlgemuth, M. Frey, and F. Gauterin. GNSSshortages-resistant and self-adaptive rear axle kinematic parameter estimator (SA-RAKPE). In IEEE Intelligent Vehicles Symposium (IV), pages 456-461. IEEE, June 2017.

[5] R. Dubé, D. Dugas, E. Stumm, J. Nieto, R. Siegwart, and C. Cadena. Segmatch: Segment based place recognition in $3 \mathrm{~d}$ point clouds. In IEEE International Conference on Robotics and Automation (ICRA), pages 5266-5272, May 2017.

[6] C. Fouque, P. Bonnifait, and D. Bétaille. Enhancement of global vehicle localization using navigable road maps and dead-reckoning. In IEEE/ION Position, Location and Navigation Symposium. IEEE, 2008.

[7] M. Harr and C. Schaefer. Robust Dead Reckoning: Calibration, Covariance Estimation, Fusion and Integrity Monitoring. Automatisiertes Fahren und vernetzte Mobilitaet (AutoReg), VDI VDE Conference, 8:91-102, January 2017.

[8] K. Lee and W. Chung. Calibration of kinematic parameters of a carlike mobile robot to improve odometry accuracy. In IEEE International Conference on Robotics and Automation, pages 2546-2551, May 2008.

[9] H. Loeliger, L. Bruderer, H. Malmberg, F. Wadehn, and N. Zalmai. On sparsity by NUV-EM, gaussian message passing, and kalman smoothing. In Information Theory and Applications Workshop (ITA), pages 1-10, Jan 2016.

[10] C. Lundquist, R. Karlsson, E. Ozkan, and F. Gustafsson. Tire radii estimation using a marginalized particle filter. IEEE Transactions on Intelligent Transportation Systems, 15(2):663-672, April 2014.

[11] R. Mur-Artal and J. D. Tardós. Orb-slam2: An open-source slam system for monocular, stereo, and rgb-d cameras. IEEE Transactions on Robotics, 33(5):1255-1262, Oct 2017.

[12] J. Rabe, M. Necker, and C. Stiller. Ego-lane estimation for lane-level navigation in urban scenarios. In IEEE Intelligent Vehicles Symposium (IV), pages 896-901, June 2016.

[13] H. E. Rauch, C. T. Striebel, and F. Tung. Maximum likelihood estimates of linear dynamic systems. AIAA Journal, 3(8):1445-1450, August 1965.

[14] R. P. D. Vivacqua, M. Bertozzi, P. Cerri, F. N. Martins, and R. F. Vassallo. Self-localization based on visual lane marking maps: An accurate low-cost approach for autonomous driving. IEEE Transactions on Intelligent Transportation Systems, 19(2):582-597, Feb 2018.

[15] F. Wadehn, L. Bruderer, J. Dauwels, V. Sahdeva, H. Yu, and H. A. Loeliger. Outlier-insensitive Kalman smoothing and marginal message passing. In 24th European Signal Processing Conference (EUSIPCO), pages 1242-1246. IEEE, August 2016.

[16] J. Zhang and S. Singh. Loam: Lidar odometry and mapping in realtime. In Robotics: Science and Systems Conference. Robotics: Science and Systems Foundation, July 2014. 